\title{
Predictive factors for exudation of quiescent choroidal neovessels detected by OCT angiography in the fellow eyes of eyes treated for a neovascular age-related macular degeneration
}

\author{
Lauriana Solecki ${ }^{1} \cdot$ Prisca Loganadane $^{1} \cdot$ Anne-Sophie Gauthier ${ }^{1} \cdot$ Manon Simonin $^{1} \cdot$ Marc Puyraveau $^{2}$. \\ Bernard Delbosc $^{1} \cdot$ Maher Saleh $^{1}$
}

Received: 24 September 2019 / Revised: 30 March 2020 / Accepted: 27 April 2020 / Published online: 12 May 2020

(c) The Author(s), under exclusive licence to The Royal College of Ophthalmologists 2020

\begin{abstract}
Background To identify predictive factors for exudation for quiescent choroidal neovessels (qCNV) in the fellow eyes of eyes treated for a neovascular age-related macular degeneration (AMD).

Methods Prospective observational study. One hundred and forty-four contralateral eyes of 144 patients treated for wet AMD were analysed. At a baseline visit, multimodal imaging including dye angiographies and optical coherence tomography angiography (OCT-A) was performed in order to detect qCNV. Patients were followed up for 12 months with a monthly assessment. The manifestation of any type of exudation (either intra- or subretinal fluid or hyperreflective subretinal material) was monitored.

Results The prevalence of qCNV in the treatment-naive eyes was $15.9 \%$ with an incidence over a 12 -month period of $2.8 \%$. In total, $40.7 \%$ of the overall neovessels remained stable with no sign of exudation, while $59.3 \%$ presented some fluid during the follow-up. A statistically significant relationship was established for the following variables preceding the exudation: increase in central macular thickness $(\mathrm{OR}=116 ; 95 \% \mathrm{CI}[4.74 ; 50530] p=0.038)$, increase in pigment epithelial detachment height $(\mathrm{OR}=1.76 ; 95 \% \mathrm{CI}[1.17 ; 3.18] p=0.021)$ and width $(\mathrm{OR}=1.53 ; 95 \% \mathrm{CI}[1.12 ; 2.62] p=0.042)$, increase in neovessels' surface on OCT-A $(\mathrm{OR}=6.32 ; 95 \% \mathrm{CI}[1.62 ; 51.0] p=0.033)$, emergence of a branching pattern $(\mathrm{OR}=7.50 ; 95 \% \mathrm{CI}[1.37 ; 61.5] p=0.032)$ and appearance of a hypointense halo surrounding the lesion $(\mathrm{OR}=10.00 ; 95 \%$ CI $[1.41 ; 206] p=0.048)$.

Conclusions The risk of exudation in the treatment-naive fellow eyes of eyes treated for neovascular AMD was notably increased in the presence of qCNV. The biomarkers identified will help to detect their activation in order to ensure prompt antiangiogenic therapy.
\end{abstract}

\section{Introduction}

Age-related macular degeneration (AMD) is a leading cause of blindness in elderly patients in industrialized countries

Supplementary information The online version of this article (https:// doi.org/10.1038/s41433-020-0936-7) contains supplementary material, which is available to authorized users.

Maher Saleh

drmahersaleh@gmail.com

1 Department of Ophthalmology, Besançon University Hospital, Besançon, France

2 Center of Clinical Methodology, Besançon University Hospital, Besançon, France
[1-4]. Two years after the onset of the disease in the first eye, a bilateralization of the neovascular form is seen in $20 \%$ of the patients, with a significant impact on the patient's quality of life [5]. Consequently, early detection of the neovascular lesion in the treatment-naive fellow eye is an essential step to start prompt antiangiogenic therapy. Recent advances in retinal imaging have significantly improved our capacity to detect such lesions. Optical coherence tomography angiography (OCT-A) is able to detect AMD-related choroidal neovessels (CNV) [6-11] with a sensitivity of $100 \%$ and a specificity of $97.5 \%$ [12]. When these neovessels are asymptomatic in treatment-naive eyes, with absence of exudation on structural OCT, they are defined as "quiescent choroidal neovessels" (qCNV) [13]. Detection of qCNV with OCT-A is also efficient, with a reported sensitivity of $81.8 \%$ and a specificity of $100 \%$ [14]. 
The management of such lesions is not yet codified and most of the authors agree not to treat them in the absence of fluid on structural OCT. Recent longitudinal studies have investigated the development of qCNV and found contradictory results. De Oliveira Dias et al. reported an increased risk of exudation in the presence of qCNV over a 12-month period [15] while Carnevali et al., found a discrepancy between the increasing size of the vascular lesion over time and the absence of exudation on OCT [16]. The objective of the current study was to identify predictive factors of qCNV exudation in treatment-naive eyes of patients treated for neovascular AMD in the fellow eye in order to improve their monitoring.

\section{Material and methods}

A prospective descriptive study was conducted at the Ophthalmology Department of the University Hospital of Besançon (France). Patients treated for a unilateral exudative AMD were enrolled. The protocol adhered to the tenets of the Declaration of Helsinki and was approved by the local ethics committee of the University Hospital of Besançon. Informed consent was obtained for each patient.

The following inclusion were applied: patients older than 50 years with unilateral exudative AMD treated with intravitreal injection of anti-vascular endothelial growth factor (VEGF). The study eye had to be naive to any treatment (i.e., intravitreal injection, photodynamic therapy, photocoagulation) with at least 6 months of previous follow-up.

The exclusion criteria were single eyes, lack of visual fixation, noncooperative patients, presence of polypoidal choroidal vasculopathy, significant media opacities, and others causes of CNV with exclusion of myopic maculopathies, angioid streaks, diabetic maculopathies, and chronic central serous retinopathy.

The clinical data collected included the estimated duration of the disease, medical history of other ophthalmologic diseases, spherical equivalent (diopters), best-corrected visual acuity (BCVA), and previous AMD treatments (including any anti-VEGF intravitreal therapy, photodynamic therapy with verteporfin, macular laser therapy).

At the time of the study, treatment protocol in the department consisted in a loading dose made of three antiVEGFs injection followed by a monthly pro re nata (PRN) protocol for most of the patients. All patients underwent a comprehensive ophthalmologic examination at the baseline visit and at each monthly follow-up visit including: BCVA measurement expressed in Early Treatment Diabetic Retinopathy Study (ETDRS) score, clinical exam at the slit lamp, fundoscopy and retinography $\left(\mathrm{EIDON}^{\circledR}\right.$, Center Vue, Italy), Spectral Domain OCT
(SD-OCT; Spectralis ${ }^{\circledast}$ Heidelberg Engineering OCT2, Heidelberg, Germany), and OCT-A (Spectralis ${ }^{\circledR}$ Heidelberg Engineering OCT2, Software $\beta$ version 6.9a, Heidelberg, Germany). Fluorescein and indocyanine green angiographies (HRA Spectralis ${ }^{\circledR}$ Heidelberg, Heidelberg, Germany) were performed at the time of diagnosis in the treated eye and repeated at least 6 months prior the inclusion. Exudative AMD eyes were treated with either ranibizumab, bevacizumab, or aflibercept according to a monthly PRN protocol. AMD staging was determined according to the classification of the Age-Related Eye Disease Study.

\section{CNV detection}

OCT-A was performed using the OCT-A module of the Spectralis ${ }^{\circledR}$ OCT2 (Software version 6.9a). The full spectrum approach enabled three-dimensional OCT-A imaging with high axial resolution $(3.9-\mu \mathrm{m} / \mathrm{pixel})$ separating thin vascular plexuses. The projection artifact removal tool was used to reduce unwanted artifacts on OCT-A images of the external retina from the superficial vascular plexus information. The raster consisted of a 512 B-scan spaced with $5.7 \mu \mathrm{m}$ providing high lateral resolution images of $512 \times 512$ from a $10 \times 10$-degree field (around $2.9 \times 2.9 \mathrm{~mm}$ ) focused on the macula. Automatic segmentation was controlled manually by the two readers and corrected if necessary, using the propagation feature (i.e., manual correction of five to seven scans enabling the automatic correction of the 512 B-scans). A slab located between the RPE and 20- $\mu$ m beneath Bruch's membrane was obtained at each visit using TruTrack Active Eye Tracking ${ }^{\text {TM }}$ to create a retinal map. The AutoRescan ${ }^{\mathrm{TM}}$ feature used this map to automatically place follow-up scans in precisely the same location as the baseline scan, thereby allowing for the monitoring of fine changes in CNV morphology and size during the follow-up study. When the acquisition was too time-consuming, the operator could switch to a high-speed mode. Images were analysed by two expert graders. Inter-reader agreement was assessed based on the set of overall OCT-A images acquired at baseline. A qCNV was defined as an asymptomatic previously untreated $\mathrm{CNV}$, without visual loss, without fundus hemorrhages, fibrosis, or Bruch's membrane break, and without intra- or subretinal fluid in two consecutive visits within 6 months [13]. In the presence of a qCNV lesion, quantitative and qualitative assessments were conducted. The area of the qCNV lesion was measured using the built-in feature of the Heidelberg navigator by two independent readers, and its location above or beneath the RPE was noted. qCNV growth was quantified in percentage of growth between the baseline visit and the end of the follow-up. The activity level of the $\mathrm{qCNV}$ was characterized according to the presence of 
branching patterns, peri-lesional hypointense halo, anastomoses, and vessel termini in the CNV [7, 14, 17-20] at each visit. Quantitative and qualitative changes on OCT-A were correlated with BCVA and exudation signs on SDOCT and monitored at each follow-up visit.

Exudation was defined as the occurrence of subretinal fluid, gray hyperreflective subretinal material (SHRM), or intraretinal cysts.

The primary outcome was the proportion of $\mathrm{qCNV}$ in OCT-A in the treatment-naive eyes. The second outcomes were the rate of occurrence of qCNV in 12-month followup, the proportion of occurrence of exudation in the study eye, and the time-lapse of occurrence of exudation.

\section{Statistical analysis}

Continuous/quantitative data are expressed as mean with standard deviation. Qualitative data are expressed as number and percentage. Continuous/quantitative data were compared via a nonparametric Mann-Whitney test, while qualitative data were compared using Fisher test's or a Chisquare test (depending on the size of the population). Interreader and inter-modality agreement were assessed using the kappa statistic. The cumulative incidence rate of exudation during the follow-up was calculated according to the Kaplan-Meier survival method and compared with a logrank test depending on the presence or the absence of $\mathrm{qCNV}$. Logistic regression analysis was performed to compare the progression of $\mathrm{qCNV}$ and search for predictive factors of exudative qCNV. The response after three intravitreal injections of anti-VEGF for patients with exudative qCNV during the follow-up was evaluated with a paired Wilcoxon test for the quantitative variables and with a paired Fisher's exact test for the qualitative variables. Statistical analyses were performed using the principle of intention to treat.

\section{Results}

In total, 144 patients with exudative AMD in one eye and treatment-naive fellow eye (study eye), were included in the study between January 2017 and January 2019 at the Ophthalmology Department of the University Hospital of Besançon (France). The patients were followed for 12 months.

\section{Baseline comparison}

The baseline characteristics of the eyes studied are summarized in Table 1. The population comprised a majority of women $(62.5 \%)$ with a mean age of $80.3( \pm 7.4)$ years. The mean follow-up at the baseline visit was 31.4 months.
Table 1 Baseline clinical characteristics of treatment-naive eyes and eyes treated for neovascular AMD.

\begin{tabular}{|c|c|c|c|}
\hline & & $\begin{array}{l}\text { Treated eye } \\
(n=144)\end{array}$ & $\begin{array}{l}\text { Treatment-naive } \\
\text { eye }(n=144)\end{array}$ \\
\hline Sex ratio $(\mathrm{M} / \mathrm{F})$ & & $54 / 90(37.5 / 62.5 \%)$ & $54 / 90(37.5 / 62.5 \%)$ \\
\hline Age (years) & & $80.3( \pm 7.4)$ & $80.3( \pm 7.4)$ \\
\hline BCVA (ETDRS) & & $61.7( \pm 17.9)$ & $76.8( \pm 9.3)$ \\
\hline CMT $(\mu \mathrm{m})$ & & $301.7( \pm 67.9)$ & $272.2( \pm 35)$ \\
\hline $\begin{array}{l}\text { Time of progression } \\
\text { (months) }\end{array}$ & & $31.4( \pm 31.1)$ & $31.4( \pm 31.1)$ \\
\hline \multirow[t]{5}{*}{ Stages of AMD } & 0 & $0(0 \%)$ & $19(13.2 \%)$ \\
\hline & 1 & $0(0 \%)$ & $28(19.4 \%)$ \\
\hline & 2 & $0(0 \%)$ & $44(30.6 \%)$ \\
\hline & 3 & $0(0 \%)$ & $27(18.7 \%)$ \\
\hline & 4 & $144(100 \%)$ & $26(18.1 \%)$ \\
\hline Atrophy & & $47(32.6 \%)$ & $26(18.1 \%)$ \\
\hline PED & & $129(90 \%)$ & $64(44.4 \%)$ \\
\hline APE & & $18(12.5 \%)$ & $36(25 \%)$ \\
\hline Serous drusen & & $10(6.9 \%)$ & $30(20.8 \%)$ \\
\hline $\begin{array}{l}\text { Reticular pseudo- } \\
\text { drusen }\end{array}$ & & $6(4.1 \%)$ & $31(21.5 \%)$ \\
\hline \multirow[t]{3}{*}{ Vitreous state } & $\begin{array}{l}\text { Complete } \\
\text { PVD }\end{array}$ & $104(72.2 \%)$ & $94(65.3 \%)$ \\
\hline & No PVD & $34(23.6 \%)$ & $41(28.5 \%)$ \\
\hline & $\begin{array}{l}\text { Vitreomacular } \\
\text { traction }\end{array}$ & $5(3.5 \%)$ & $9(6.2 \%)$ \\
\hline \multirow[t]{4}{*}{ Type of CNV } & 1 & $64(44.4 \%)$ & - \\
\hline & 2 & $61(42.4 \%)$ & - \\
\hline & $1+2$ & $14(9.7 \%)$ & - \\
\hline & 3 & $5(3.5 \%)$ & - \\
\hline \multirow[t]{3}{*}{ Injected molecule } & Ranibizumab & $81(56.3 \%)$ & - \\
\hline & Aflibercept & $46(31.9 \%)$ & - \\
\hline & Bevacizumab & $17(11.8 \%)$ & - \\
\hline $\begin{array}{l}\text { Number of } \\
\text { intravitreal } \\
\text { injections }\end{array}$ & & $18( \pm 15.6)$ & - \\
\hline
\end{tabular}

$B C V A$ best-corrected visual acuity, $C M T$ central macular thickness, $P E D$ pigment epithelial detachment, $A P E$ alteration of pigment epithelium, $P V D$ posterior vitreous detachment, $C N V$ choroidal neovessel.

The mean BCVA in treatment-naive eyes was $76.8( \pm 9.3)$ ETDRS letters. These eyes were affected by various stages of AMD. OCT showed atrophy in $18.1 \%$, pigment epithelial detachment (PED) in $44.4 \%$, presence of serous drusen in $20.8 \%$, and/or reticular pseudo-drusen in $21.5 \%$ of cases.

At baseline, qCNV were detected in 23 (15.9\%) treatment-naive eyes. Eyes with qCNV $(\mathrm{qCNV}+)$ and eyes without $\mathrm{qCNV}(\mathrm{qCNV}-)$ were similar in term of sex ratio, age, BCVA, CMT, time of progression of AMD, and vitreous status. There were significantly more $\mathrm{qCNV}+$ eyes with stage $2 \mathrm{a}$ AMD ( $48 \%$ vs. $24 \%, p=0.037$ ). OCT features such as PED, serous drusen, and reticular pseudodrusen were also significantly more frequent in $\mathrm{qCNV}+$ eyes than in qCNV - eyes (respectively, $p<0.001, p<0.01$, and $p=0.026$ ). (Supplementary material 1 ). 
Fig. 1 Flow chart of the study. Quiescent choroidal neovessel $(\mathrm{qCNV}+)$. Eyes without quiescent choroidal neovessels $(\mathrm{qCNV}-$ ). First row corresponds to the baseline visit and last row to the last follow-up visit. Three eyes with no qCNV at baseline developed $\mathrm{CNV}$ related exudation during follow-up.

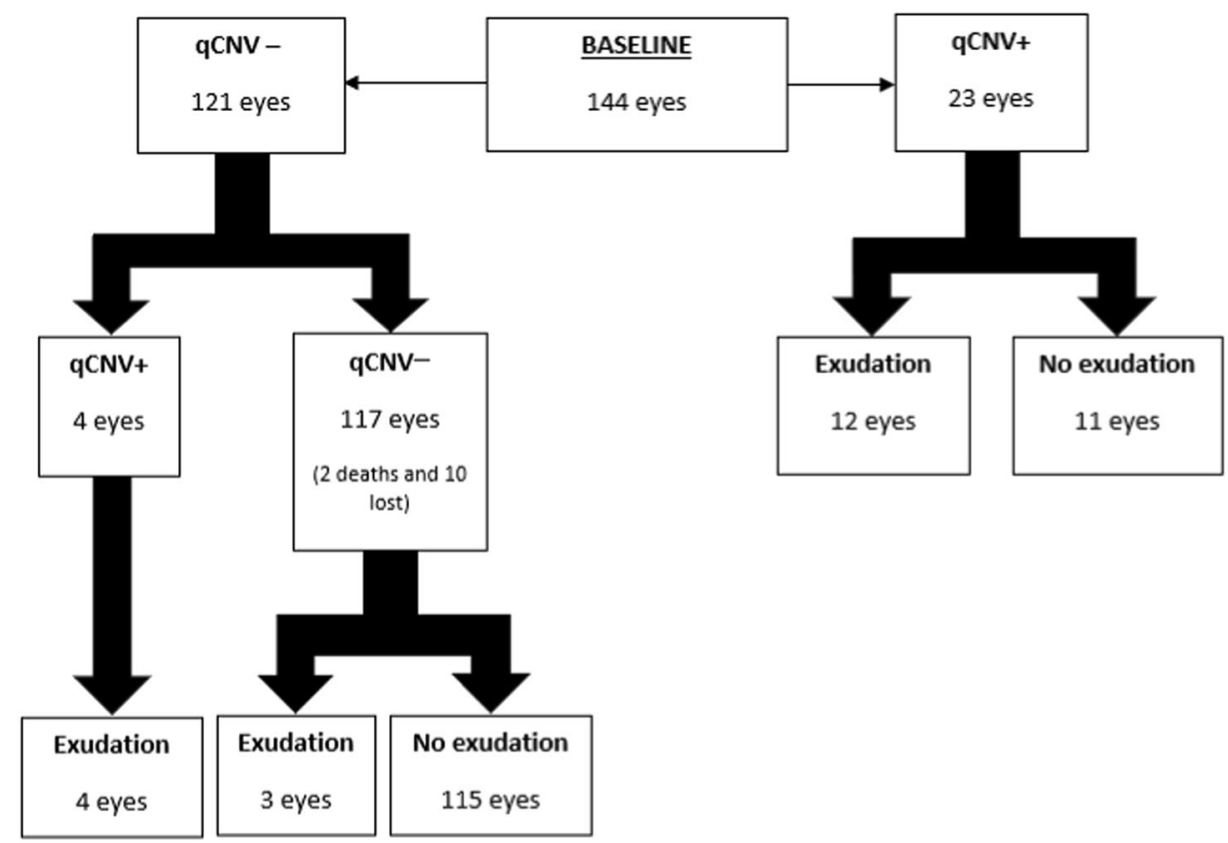

\section{Follow-up period}

Among 144 patients, 12 patients did not reach the 12-month endpoint: two died, and ten were lost to follow-up. During the follow-up period, qCNV appeared in four eyes (2.8\%). The overall incidence of exudation in the non-treated eyes was $13.2 \%$ with $59.3 \%$ of the qCNV+ eyes at baseline showing exudation during the follow-up (vs. $2.7 \%$ in the qCNV - group) (Fig. 1).

According to the log-rank test results, the occurrence of the exudation was significantly greater in $\mathrm{qCNV}+$ eyes than in $\mathrm{qCNV}-$ eyes $(p<0.0001)$ (Supplementary material 2 ). Therefore, the relative risk of exudation at 12 months was significantly increased in $\mathrm{qCNV}+$ eyes $(\mathrm{RR}=23.1 ; 95 \% \mathrm{CI}$ [7.66; 18.72]).

The interval to exudation from the baseline visit was 5.8 $( \pm 4.2)$ months on average for the $\mathrm{qCNV}+$ eyes vs. 4.3 $( \pm 3.4)$ months for the qCNV- eyes $(p=0.57)$.

\section{Comparison between activated qCNV + and those remaining quiescent}

No statistical difference was observed in terms of gender, age, baseline BCVA, time of progression of the disease, vitreous status, or OCT features between exudative and non-exudative $\mathrm{qCNV}+$. (Supplementary materials 3 and 4 ). However, the hypointense halo around the $\mathrm{qCNV}+$ was significantly more frequent in exudative $\mathrm{qCNV}+$ eyes than in non-exudative $\mathrm{qCNV}+$ eyes $(58 \%$ vs. $9.1 \% p=0.027)$.

The ocular characteristics were compared between the baseline visit and the visit preceding exudation or the end of the follow-up if no exudation was noticed. In univariate analysis, a decrease in visual acuity was observed in exudative $\mathrm{qCNV}+(-4.75$ letters on average vs. -0.2 letters in non-exudative $\mathrm{qCNV}+, p=0.013)$. The CMT increased significantly $(16.9 \%$ vs. $-3.2 \% p<0.001)$ as did the height and the width of the PED (respectively $71.3 \%$ vs. $0.03 \%$ $p<0.01$ and $101 \%$ vs. $-7.59 \% p<0.01$ ) (Supplementary material 5). The neovascular lesion was also significantly enlarged $(+250 \%$ vs. $+22.6 \% p<0.01)$ (Supplementary material 5). The appearance of a branching pattern and the formation of a hypointense halo around the neovascular lesion often preceded fluid formation (Fig. 2) (respectively $62 \%$ vs. $18 \%, p=0.047$ and $50 \%$ vs. $9.1 \%, p=0.042$ ) (Table 2).

The logistic regression analysis also pointed out the relationship between the following features of previously quiescent $\mathrm{CNV}$ and exudation: visual loss, increase in CMT, increase in PED size, lesion enlargement, occurrence of a branching pattern, and manifestation of a hypointense halo were related to the occurrence of exudation at some point during the follow-up. Thus, a reduction of the BCVA was associated with a higher risk of exudation $(\mathrm{OR}=0.69$; $95 \%$ CI $[0.466 ; 0.906] p=0.024)$. A rise of $10 \%$ of the CMT was related to an increased risk of exudation $(\mathrm{OR}=$ $116 ; 95 \%$ CI $[4.74 ; 50530] p=0.038)$. An increase of $10 \%$ in PED height and width was also associated with a higher risk of exudation $(\mathrm{OR}=1.76 ; 95 \% \mathrm{CI}[1.17 ; 3.18] p=$ 0.021 and $\mathrm{OR}=1.53 ; 95 \%$ CI $[1.12 ; 2.62] p=0.042$, respectively). A doubling in lesion surface was linked to a 6.32-fold risk of exudation (95\% CI $[1.62 ; 51.0] p=0.033$ ). The occurrence of a branching pattern of the qCNV and the manifestation of a hypointense halo (Fig. 2) increased the risk of exudation by 7.5 - and 10-fold, respectively (95\% CI 
Fig. $2 \mathrm{qCNV}+$ at the time of diagnosis and at the 11- and 12-month follow-up, when subretinal fluid was detected. A hypointense halo preceded fluid formation on OCT-A and lasted until the final endpoint.
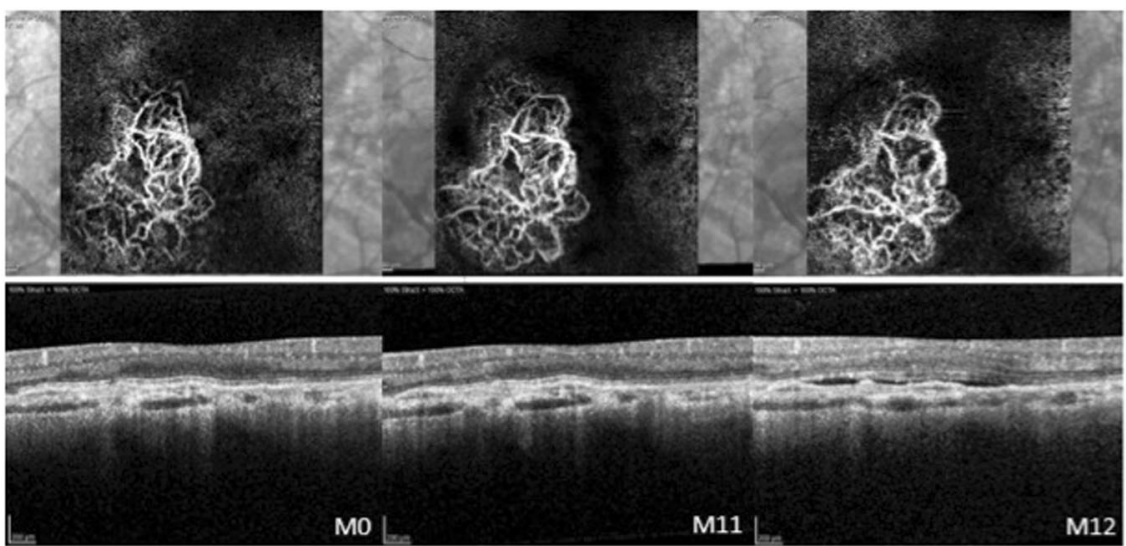

Table 2 Comparison of the clinical and OCT characteristics of exudative $\mathrm{qCNV}+$ eyes $(\mathrm{ex}+)$ and non-exudative $\mathrm{qCNV}+$ eyes (ex-) (univariate analysis) between the baseline visit and the visit preceding exudation.

\begin{tabular}{lccr}
\hline & $\mathrm{qCNV}+\mathrm{ex}-(n=11)$ & $\mathrm{qCNV}+\mathrm{e}+(n=16)$ & \multicolumn{1}{c}{$p$} \\
\hline Variation of the BCVA (ETDRS) & $-0.182( \pm 3.19)$ & $-4.75( \pm 5.26)$ & $\mathbf{0 . 0 1 3}$ \\
Variation of the CMT $(\%)$ & $-3.20( \pm 4.82)$ & $16.9( \pm 26.4)$ & $<\mathbf{0 . 0 0 1}$ \\
Variation of the height of the PED $(\%)$ & $0.03( \pm 18.4)$ & $71.3( \pm 118)$ & $<\mathbf{0 . 0 1}$ \\
Variation of the width of the PED $(\%)$ & $-7.59( \pm 26.6)$ & $101( \pm 137)$ & $<\mathbf{0 . 0 1}$ \\
Variation of the qCNV surface $(\%)$ & $22.6( \pm 49.4)$ & $250( \pm 317)$ & $<\mathbf{0 . 0 1}$ \\
Occurrence of anastomoses & $1(9.1 \%)$ & $5(31 \%)$ & 0.35 \\
Appearance of a branching pattern & $2(18 \%)$ & $10(62 \%)$ & $\mathbf{0 . 0 4 7}$ \\
Manifestation of a hypointense halo & $1(9.1 \%)$ & $8(50 \%)$ & $\mathbf{0 . 0 4 2}$ \\
Appearance of vessel termini & $1(9.1 \%)$ & $3(19 \%)$ & 0.62 \\
\hline
\end{tabular}

$B C V A$ best-corrected visual acuity, $C M T$ central macular thickness, $P E D$ pigment epithelial detachment.

Bold values indicate statistical significance $p<0.05$.
$[1.37 ; 61.5] p=0.032$ and $95 \%$ CI $[1.41 ; 206] p=0.048)$. (Supplementary material 6).

\section{Treatment response}

A mean gain of +2.9 letters was observed after the loading dose constituted of three monthly intravitreal anti-VEGF injections $(p=0.033)$ delivered to a previous quiescent $\mathrm{CNV}$ that had now become exudative. A reduction in central macular thickness of $-98.4 \mu \mathrm{m}$ on average was also seen $(p<0.001)$. The PED size decreased $(p<0.01$ for height and $p=0.02$ for width). However, the lesion's area and other OCT-A features remained unchanged or even worsened under treatment. (Fig. 3 and Supplementary materials 7 and 8).

\section{Discussion}

Recent studies have found a prevalence of $\mathrm{qCNV}$ detected by OCT-A ranging from 1.58 to $27 \%[15,16,21,22]$. In the current study, the prevalence was $15.9 \%$ with a 12 -month incidence of $2.8 \%$. This result is in accordance with the De
Oliveira et al. study that included 160 patients and found a $14.4 \%$ prevalence and a $5.4 \%$ incidence [15]. The presence of qCNV increased the risk of exudation by 23.1 -fold. The predictive factors of fluid formation were loss of visual acuity, increase in the retinal thickness, PED enlargement on structural OCT, increase in the lesion's surface, and the manifestation of specific features on OCT-A such a branching pattern, and/or a hypointense halo surrounding the lesion. Most of the lesions were type 1 neovascular lesions, as reported previously [13, 15, 21, 23-25]. The majority of qCNV were located in a PED (83\%) that was totally or partially hyperreflective $(94.7 \%)$. Monitoring the size of the PED was of great value in detecting the transformation of qCNV to active lesions. Indeed, a $10 \%$ increase in height or width was associated with an increased risk of exudation of 1.76 and 1.53 , respectively. Previous studies reported, the presence of an irregular PED with hyperreflective content in cases of qCNV but none correlated PED growth with the risk of exudation $[13,21,22,24]$.

While OCT-A allows for a more precise assessment of the lesion surface than ICG angiography, few quantitative longitudinal data have been reported with this technique. 
Fig. 3 Progression of a $\mathrm{qCNV}+$ lesion before (left) and after the loading dose made of three monthly intravitreal antiVEGF injections (right). OCTA features and size of the lesion remained unchanged (top), while reduction in PED size and disappearance of the subretinal fluid were observed on structural OCT (bottom).

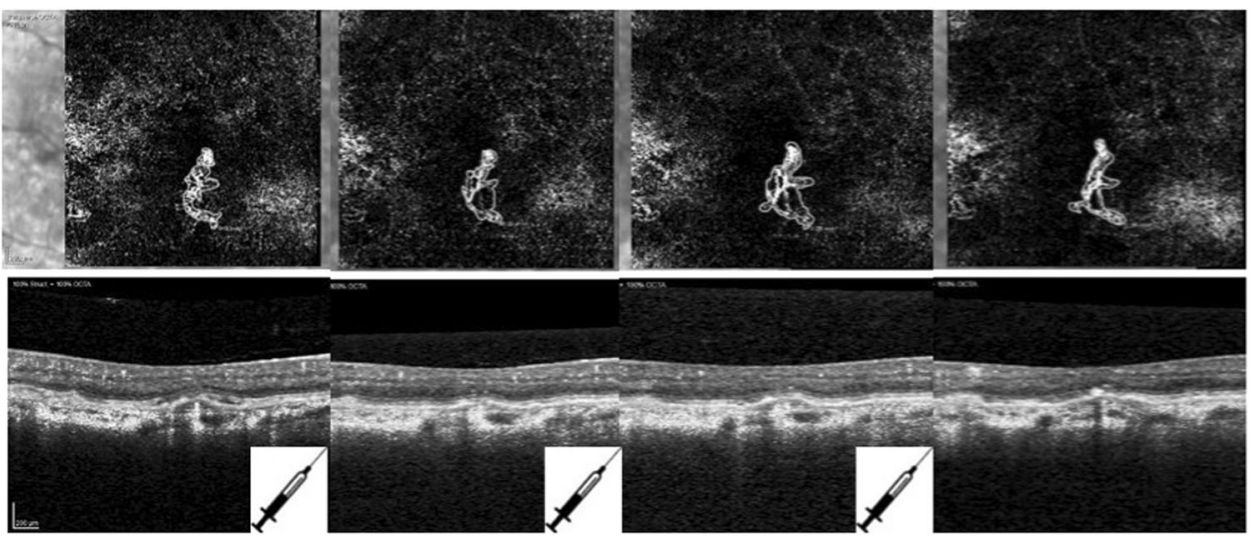

For instance, Querques et al. found a stable lesion over time [13], whereas, we observed a growth in some of the neovascular lesions. A doubling in size was associated with a sixfold increase in risk of exudation. We also applied the description of qCNV proposed by Coscas et al. [7, 20] and found that the occurrence of a branching pattern and a hypointense halo around the lesion were also associated with a higher risk of exudation by 4.55- and 10-fold, respectively. Dark halos have been frequently observed in active CNV lesions [7, 26] and could be explained by a vascular steal by the growing lesion or a secondary decrease in neighboring choriocapillary density (Supplementary material 9).

Treatment response was comparable with the results obtained in non-quiescent $\mathrm{CNV}$ with an improvement of BCVA and a decrease in retinal thickness. The gain in visual acuity after three anti-VEGF intravitreal injections may be seen as limited, but it should be put into perspective with the relatively high level of baseline visual acuity. Interestingly, the lesion did not shrink despite prompt treatment, as shown for neovascular AMD [27], pointing to the failure of the current therapies to induce a reduction in lesion size rather than the stage of the disease at the time of treatment, since a neovessel enlargement was seen in newly formed lesion treated with anti-VEGF. This study has a number of drawbacks. OCT-A is a relatively new technology in the clinical field and is subject to continuous improvement. However, most upgrades in image treatment such as artifact removal or propagation of manual corrections could be applied to previous acquisitions, thus facilitating the comparison of images set over the study period. Moreover, the neovascular AMD eyes were treated with different anti-VEGF molecules, but this should not affect the natural history of quiescent lesions in the fellow study eye. Furthermore, most patients were not enrolled at the primary diagnosis or the primary treatment of AMD of the affected eyes. Thereby, we can wonder about the duration of evolution for the revealed qCNV. A longer follow-up will also be interesting to assess other factors such as progression of, geographic atrophy or photoreceptor loss in eyes with qCNV.

In conclusion, the prevalence of qCNV in this study was $15.9 \%$. The presence of qCNV was associated with a higher risk of exudation. A decrease in visual acuity, increase in retinal thickness, increase in PED size, enlargement of the qCNV on OCT-A, or the manifestation of a branching pattern or a dark halo are predictive signs of exudation that should be looked for during the monitoring of these lesions at high risk of activation.

\section{Summary}

\section{What was known before:}

- When the choroidal neovessels in AMD are asymptomatic in treatment-naive eyes, with absence of exudation on structural OCT, they are defined as "quiescent choroidal neovessels" by Querques et al.

\section{What this study adds:}

- The risk of exudation in the treatment-naive eyes of eyes treated for neovascular AMD was notably increased in the presence of qCNV. The predictive signs of exudation were identified. They should be looked for during the monitoring of these lesions at high risk of activation.

Acknowledgements This work was funded by the Francomtoise Association for Research in Ophthalmology (AFCRO, Besançon, France). The authors declare their independence in reporting the data.

\section{Compliance with ethical standards}

Conflict of interest LS, PL, ASG, MS, and MP: None. BD: Consultant (Alcon, Théa, Allergan), MS: Consultant (Novartis, Bayer, Allergan). 
Publisher's note Springer Nature remains neutral with regard to jurisdictional claims in published maps and institutional affiliations.

\section{References}

1. Augood CA, Vingerling JR, de Jong PTVM, Chakravarthy U, Seland J, Soubrane G, et al. Prevalence of age-related maculopathy in older Europeans: the European Eye Study (EUREYE). Arch Ophthalmol. 2006;124:529-35.

2. Friedman DS, O'Colmain BJ, Muñoz B, Tomany SC, McCarty C, de Jong PTVM, et al. Prevalence of age- related macular degeneration in the United States. Arch Ophthalmol. 2004;122:564-72.

3. Wong WL, Su X, Li X, Cheung CMG, Klein R, Cheng C-Y, et al. Global prevalence of age-related macular degeneration and disease burden projection for 2020 and 2040: a systematic review and meta-analysis. Lancet Glob Health. 2014;2:e106-e116.

4. Ferris FL, Fine SL, Hyman L. Age-related macular degeneration and blindness due to neovascular maculopathy. Arch Ophthalmol. 1984;102:1640-2.

5. Chopra R, Wagner SK, Fasler K, Kortuem KU, Pontikos N, Afshar F, et al. Development of neovascular age-related macular degeneration in fellow eyes of patients undergoing intravitreal anti-VEGF therapy at a large tertiary ophthalmic hospital. Investig Ophthalmol Vis Sci. 2018;59:816-816.

6. Jia Y, Bailey ST, Wilson DJ, Tan O, Klein ML, Flaxel CJ, et al. Quantitative optical coherence tomography angiography of choroidal neovascularization in age-related macular degeneration. Ophthalmology. 2014;121:1435-44.

7. Coscas GJ, Lupidi M, Coscas F, Cagini C, Souied EH. Optical coherence tomography angiography versus traditional multimodal imaging in assessing the activity of exudative age-related macular degeneration: a new diagnostic challenge. Retina. 2015;35: 2219-28.

8. Spaide RF, Fujimoto JG, Waheed NK, Sadda SR, Staurenghi G. Optical coherence tomography angiography. Prog Retin Eye Res. 2018;64:1-55.

9. Roisman L, Goldhardt R. OCT angiography: an upcoming noninvasive tool for diagnosis of age-related macular degeneration. Curr Ophthalmol Rep. 2017;5:136-40.

10. Nagiel A, Sadda SR, Sarraf D. A promising future for optical coherence tomography angiography. JAMA Ophthalmol. 2015;133:629-30.

11. de Carlo TE, Romano A, Waheed NK, Duker JS. A review of optical coherence tomography angiography (OCTA). Int J Retin Vitreous. 2015;1:5.

12. Faridi A, Jia Y, Gao SS, Huang D, Bhavsar KV, Wilson DJ, et al. Sensitivity and specificity of OCT angiography to detect choroidal neovascularization. Ophthalmol Retin. 2017;1:294-303.

13. Querques G, Srour M, Massamba N, Georges A, Ben Moussa N, Rafaeli $\mathrm{O}$, et al. Functional characterization and multimodal imaging of treatment-naive 'quiescent' choroidal neovascularization. Investig Ophthalmol Vis Sci. 2013;54:6886-92.

14. Carnevali A, Cicinelli MV, Capuano V, Corvi F, Mazzaferro A, Querques L, et al. Optical coherence tomography angiography: a useful tool for diagnosis of treatment-naïve quiescent choroidal neovascularization. Am J Ophthalmol. 2016;169:189-98.

15. de Oliveira Dias JR, Zhang Q, Garcia JMB, Zheng F, Motulsky $\mathrm{EH}$, Roisman L, et al. Natural history of subclinical neovascularization in nonexudative age-related macular degeneration using swept-source OCT angiography. Ophthalmology. 2018; 125:255-66.

16. Carnevali A, Sacconi R, Querques L, Marchese A, Capuano V, Rabiolo A, et al. Natural history of treatment-naïve quiescent choroidal neovascularization in age-related macular degeneration using oct angiography. Ophthalmol Retin. 2018;2:922-30.

17. Kuehlewein L, Bansal M, Lenis TL, Iafe NA, Sadda SR, Bonini Filho MA, et al. Optical coherence tomography angiography of type 1 neovascularization in age-related macular degeneration. Am J Ophthalmol. 2015;160:739-48.e2.

18. El Ameen A, Cohen SY, Semoun O, Miere A, Srour M, QuarantaEl Maftouhi M, et al. TYPE 2 neovascularization secondary to age-related macular degeneration imaged by optical coherence tomography angiography. Retina. 2015;35:2212-8.

19. Miere A, Querques G, Semoun O, El Ameen A, Capuano V, Souied EH. Optical coherence tomography angiography in early type 3 neovascularization. Retina. 2015;35:2236-41.

20. Al-Sheikh M, Iafe NA, Phasukkijwatana N, Sadda SR, Sarraf D. Biomarkers of neovascular activity in age-related macular degeneration using optical coherence tomography angiography. Retina. 2018;38:220-30.

21. Roisman L, Zhang Q, Wang RK, Gregori G, Zhang A, Chen C-L, et al. Optical coherence tomography angiography of asymptomatic neovascularization in intermediate age-related macular degeneration. Ophthalmology. 2016;123:1309-19.

22. Palejwala NV, Jia Y, Gao SS, Liu L, Flaxel CJ, Hwang TS, et al. Detection of non-exudative choroidal neovascularization in agerelated macular degeneration with optical coherence tomography angiography. Retina. 2015;35:2204-11.

23. Capuano V, Miere A, Querques L, Sacconi R, Carnevali A, Amoroso F, et al. Treatment-naïve quiescent choroidal neovascularization in geographic atrophy secondary to nonexudative age-related macular degeneration. Am J Ophthalmol. 2017:182:45-55.

24. Nehemy MB, Brocchi DN, Veloso CE. Optical coherence tomography angiography imaging of quiescent choroidal neovascularization in age-related macular degeneration. Ophthalmic Surg Lasers Imaging Retin. 2015;46:1056-7.

25. Schneider U, Gelisken F, Inhoffen W, Kreissig I. Indocyanine green angiographic findings in fellow eyes of patients with unilateral occult neovascular age-related macular degeneration. Int Ophthalmol. 1997;21:79-85.

26. Rispoli M, Savastano MC, Lumbroso B. Quantitative vascular density changes in choriocapillaris around CNV after Anti-VEGF treatment: dark halo. Ophthalmic Surg Lasers Imaging Retin. 2018;49:918-24

27. Xu D, Dávila JP, Rahimi M, Rebhun CB, Alibhai AY, Waheed $\mathrm{NK}$, et al. Long-term progression of type 1 neovascularization in age-related macular degeneration using optical coherence tomography angiography. Am J Ophthalmol. 2018;187:10-20. 